\title{
Identification of a Heat-Inducible Element of Cysteine Desulfurase Gene Promoter in Lentinula edodes
}

\author{
Zhicheng Huang ${ }^{1}$, Xiaoyu Lei ${ }^{1}$, Xi Feng ${ }^{2}$, Shuangshuang Gao ${ }^{1}$, Gangzheng Wang ${ }^{3}$, \\ Yinbing Bian ${ }^{3}$, Wen Huang ${ }^{1}$ and Ying Liu ${ }^{1} * \mathbb{D}$ \\ 1 College of Food Science and Technology, Huazhong Agricultural University, Wuhan 430070, China; \\ HuangZhiCheng1210@163.com (Z.H.); xiaoyulei1988@126.com (X.L.); 13720161459@163.com (S.G.); \\ huangwen@mail.hzau.edu.cn (W.H.) \\ 2 Department of Nutrition, Food Science and Packaging, California State University, San Jose, CA 95192, USA; \\ xi.feng@sjsu.edu \\ 3 Institute of Applied Mycology, Plant Science and Technology College, Huazhong Agricultural University, \\ Wuhan 430070, China; wgzhau@163.com (G.W.); bianyinbing@mail.hzau.edu.cn (Y.B.) \\ * Correspondence: yingliu@mail.hzau.edu.cn; Tel.: +86-13407161906
}

Received: 25 April 2019; Accepted: 13 June 2019; Published: 14 June 2019

\begin{abstract}
Volatile organosulfur compounds are the main components that contribute to the unique aroma of dried Lentinula edodes. They are mainly generated during the hot-air drying process, and cysteine desulfurase is the key enzyme in this process. Temperature may be an essential factor of volatile organosulfur compound production by influencing the expression of the cysteine desulfurase gene. In this study, the promoter sequence of the cysteine desulfurase gene $(p C S)$ was cloned and analyzed using bioinformatics tools. A series of 5 'deletion fragments and site-directed mutations of $p C S$ were constructed to identify the element that responds to heat stress. Six heat shock transcription factor (HSTF) binding sites were predicted by SCPD (The Promoter Database of Saccharomyces cerevisiae) and three of the binding sites were predicted by Yeastract (Yeast Search for Transcriptional Regulators and Consensus Tracking) in $p C S$. The results indicated that $p C S$ was able to drive the expression of the EGFP (Enhanced Green Fluorescent Protein) gene in L. edodes. Moreover, the fluorescence intensity increased after heat stress. The changes in fluorescence intensity of different $5^{\prime}$ deletion fragments showed that the heat response region was located between $-500 \mathrm{bp}$ and $-400 \mathrm{bp}$ in $p C S$. The site-directed mutation analysis further showed that the heat-inducible element was between $-490 \mathrm{bp}$ and $-500 \mathrm{bp}$ (TTTCTAGAAT) in $p C S$. Our results provide molecular insight for studying the formation of volatile organosulfur compounds in dried L. edodes.
\end{abstract}

Keywords: Lentinula edodes; gene promoter; volatile organosulfur compounds; heat-inducible element

\section{Introduction}

Lentinula edodes (shiitake mushroom), the second most cultivated edible mushroom in the world, has been used as food and traditional medicine for 2000 years [1]. Fresh mushrooms usually rapidly go through open-caps, browning or shrinking after harvesting, which cause the loss of commodity value and edible quality. Fresh mushrooms are often dried to preserve their quality and nutritional value. Moreover, the smell of dried L. edodes is one of the most important indexes to assess its quality, which is entirely different from fresh L. edodes. Therefore, dried L. edodes is especially prized because of its characteristic flavor [2]. Sulfur compounds including disulfide, trisulfide, 1,2,4-trithiolane, 1,2,4,6-tetrathione, and 1,2,3,5,6-pentathiepane (lenthionine) contribute the major flavors of dried L. edodes [3], while carbon-8 compounds are the major volatile compounds in fresh L. edodes [4]. Lenthionine, a cyclic sulfur compound, is the characteristic flavor component in dried L. edodes due to its low threshold value $[5,6]$. 
However, different drying methods significantly affect the flavor of dried L. edodes. Previous studies in our lab also showed that the volatile sulfide is significantly different during three drying methods including natural drying, hot-air drying, and freeze drying. Convective drying at $80^{\circ} \mathrm{C}$ for $120 \mathrm{~min}$ has the highest contents of total volatiles and cyclic sulfur compounds [4,7]. Among all of the drying methods, hot-air drying is the most economical and suitable to produce dried L. edodes at the industrial scale. However, the mechanisms of drying temperature on the formation of volatile organosulfur compounds of $L$. edodes is still unknown.

Lentinic acid ( $\gamma$-L-glutamyl-cysteine sulfoxide) is the precursor of lenthionine, and $\gamma$-glutamyl transpeptidase (GGT) and cysteine sulfoxide lyase (C-S lyase) are the two key enzymes in this reaction $[8,9]$. The content of lentinic acid increased significantly after a drying process at $40{ }^{\circ} \mathrm{C}$ for $4 \mathrm{~h} \mathrm{[10].} \mathrm{Recently,} \mathrm{the} \mathrm{C-S} \mathrm{lyase} \mathrm{in} \mathrm{L.} \mathrm{edodes} \mathrm{was} \mathrm{reported} \mathrm{to} \mathrm{be} \mathrm{a} \mathrm{novel} \mathrm{cysteine} \mathrm{desulfurase} \mathrm{and} \mathrm{not} \mathrm{a}$ type of cysteine sulfoxide lyase [11]. An exogenous expression system of the cysteine desulfurase gene (Csl) was constructed, and it obtained an active recombinant enzyme, which was found to catalyze lentinic acid to generate volatile organosulfur compounds [12]. However, there are few studies on the molecular function of cysteine desulfurase on the formation of volatile organosulfur compounds in L. edodes. In addition, the temperature may influence the formation of volatile organosulfur compounds in L. edodes by regulating the transcription level of the cysteine desulfurase gene during the drying process [13].

The heat shock element (HSE) is a specific sequence in the promoter region that binds with the heat shock transcription factor (HSTF/Hsf1) and promotes the transcription level of heat-inducible genes during the thermal resistance process. The HSE is a functional domain present in many genes such as APX1 [14], HSP [15], PGK [16], and heat shock operon danK [17]. Thus, HSE or HSE-like elements may be the most important factors for the gene $C s l$ to respond to heat stress during the hot-air drying process. The objectives of this study were to analyze the function of the promoter of Csl gene $(p C S)$ in L. edodes.

\section{Results}

\subsection{Bioinformatic Analysis of Putative Cis-Elements in pCS}

The bioinformatics analysis showed that some putative elements were predicted in $p C S$. As shown in Table 1, 44 of elements in $p C S$ were predicted by SCPD. An RNA polymerase II binding site TATA-Box (TATATA) was found to be located $57 \mathrm{bp}$ upstream of the ATG sequence on both strands. More importantly, seven elements with homology to heat shock transcription factor (HSTF) binding sites (TTCAACGAA) were found which meant the gene Csl may be able to respond to heat stress. The $p C S$ also contained one $\mathrm{Cu}^{2+}$ response element (GAGCAAA, -664), one element involved in DNA repair (CTTCCT, -1282), and one RNA polymerase I biding site (CCACCCG, -19). As shown in Table 2, 77 of the elements in $p C S$ were predicted by Yeastract, and 43 of the elements were on the forward strand, whereas the others were on the opposite strand. Three of the heat shock factor (Hsf1) binding sites were found on both strands. Moreover, the positions of Hsf1 were consistent with three of the HSTF binding sites. A $\mathrm{Cu}^{2+}$ response element (TTTGCKCR, -636) also existed, while its position and the signal consensus in $p C S$ were different compared with the results of the SCPD. Nine of the Gcr1p binding sites were predicted in $p C S$, whereas the Gcr1p transcriptional factor was involved in the regulation of glycolytic genes [18]. There were 27 of the predicted transcriptional binding sites, including nine of the Mpt3p, one of the Skn7p, 15 of the Stb5p, and two of the Yap1p which participated in oxidative stress. Tec1 $\mathrm{p}$ was reported to be a transcription factor involved in the expression of hypha-specific genes in Candida albicans [19]. Two of Tec1 $\mathrm{p}$ binding sites were predicted in $p C S$. Totally, three of the heat shock transcription factors binding sites were predicted by both Yeastract and SCPD, and the binding sites were located between $-801 \mathrm{bp}$ and $-811 \mathrm{bp},-522 \mathrm{bp}$ and $-512 \mathrm{bp}$, and $-490 \mathrm{bp}$ and $-500 \mathrm{bp}$. 
Table 1. The transcription factor binding sites predicted by SCPD.

\begin{tabular}{cccc}
\hline Element Name & Signal Sequence & Putative Function & Numbers \\
\hline GCN4 & TGAATA & Transcriptional activator binding site & 17 \\
HSTF & TTCAACGAA & Involved in heat response & 7 \\
REB1 & CCACCCG & RNA polymerase Ilbinding site & 1 \\
ECB & GGAAAAA & Early cell-cycle box element & 1 \\
ADR1 & TCTCC & Transcriptional activator binding site & 3 \\
GCR1 & CTTCC & Involved in decomposition of sugar & 7 \\
CuRE & GAGCAAA & Cu $^{2+}$ response element & 1 \\
PHO4 & CACGTT & Activation of phosphate metabolism related genes & 2 \\
ABF1 & TCATTCCAGACG & Transcriptional activation of numerous genes & 1 \\
TBP & TATATA & TATA-Box binding protein & 2 \\
UASPHR & CTTCCT & Involved in DNA repair & 1 \\
STRE & AGGGG & Involved in stress response & 1 \\
\hline
\end{tabular}

Table 2. The transcription factor binding sites predicted by Yeastract.

\begin{tabular}{|c|c|c|c|}
\hline Element Name & Signal Sequence & Putative Function & Numbers \\
\hline Cat8, Sip4 & NCCDTYNVNCCNG & Involved in the rearrangement of carbon metabolism & 1 \\
\hline Fkh1, Fkh2 & RYMAAYA & Involved in cell cycle and differentiation & 3 \\
\hline Gat1, Gln3, Gzf3 & GATAAG & Involved in glyceride metabolism & 1 \\
\hline Gen 4 & TGATTCA & Activating amino acid synthesis related genes & 1 \\
\hline Hsf1 & NTTCNNGAAN & HSTF binding site & 6 \\
\hline Mac1 & TTTGCKCR & $\mathrm{Cu}^{2+}$ response element & 1 \\
\hline Mot3 & AAGGWT & Involved in oxygen stress & 8 \\
\hline Msn2, Msn4, Rph1 & CCCTC & Involved in stress response & 1 \\
\hline Skn7 & GGCCAGA & Response to oxidative stress and osmoregulation & 1 \\
\hline Stb5 & CGGNS & Regulating multidrug resistance and oxidative stress response & 15 \\
\hline Tec1 & CATTCT & Regulating hyphal growth & 2 \\
\hline $\mathrm{Xbp} 1$ & CTCGA & Cyclin gene transcriptional repression & 2 \\
\hline Yap1 & TGACAA & Required for oxidative stress & 2 \\
\hline Rim101 & TGCCAAG & Response to $\mathrm{pH}$ and in cell wall construction & 2 \\
\hline Haa1 & SMGGSG & Involved in adaptation to weak acid stress & 3 \\
\hline Com2 & ATAGGGT & Involved in adaptation to stress & 1 \\
\hline
\end{tabular}

\subsection{EGFP Expression in Transformants Driven by Full-Length $p$ CS}

After being grown on MYG(Malt extract glucose) medium for 3 days, 13 of the transformants which expressed EGFP under Csl promoter were identified via PCR reaction with primers GPD-F/R. Three of the identified transformants named pCS-D0-1, pCS-D0-3, and pCS-D0-5 were selected for analysis using an OLYMPUS BX51 fluorescence microscope. The fluorescence intensity of each transformant was calculated and given by Image-Pro Express. As shown in Figure 1a, the wild-type strain W1 mycelia showed a weak fluorescence under $25^{\circ} \mathrm{C}$. However, the fluorescence intensity of the three transformants was two-fold higher than that of W1. Our results indicated that the promoter $p C S$ were able to drive the expression of EGFP in L. edodes.

After heat stress, the fluorescence intensity of pCS-D0 transformants significantly increased, whereas the fluorescence intensity of W1 was not changed (Figure 2d,f). Meanwhile, the relative expression level of Csl in wild-type W1 showed three-fold higher afterheat stress (Figure 1b). Usually, a constitutive promoter such as the GPD (glyceraldehyde-3-phosphate dehydrogenase) promoter can drive gene expression at a high level under any stimuli. However, the promoter $p C S$ showed low 
activity at room temperature, while its activity increased after heat stress. Thus, the promoter $p C S$ is suggested to be a heat-inducible promoter in L. edodes.
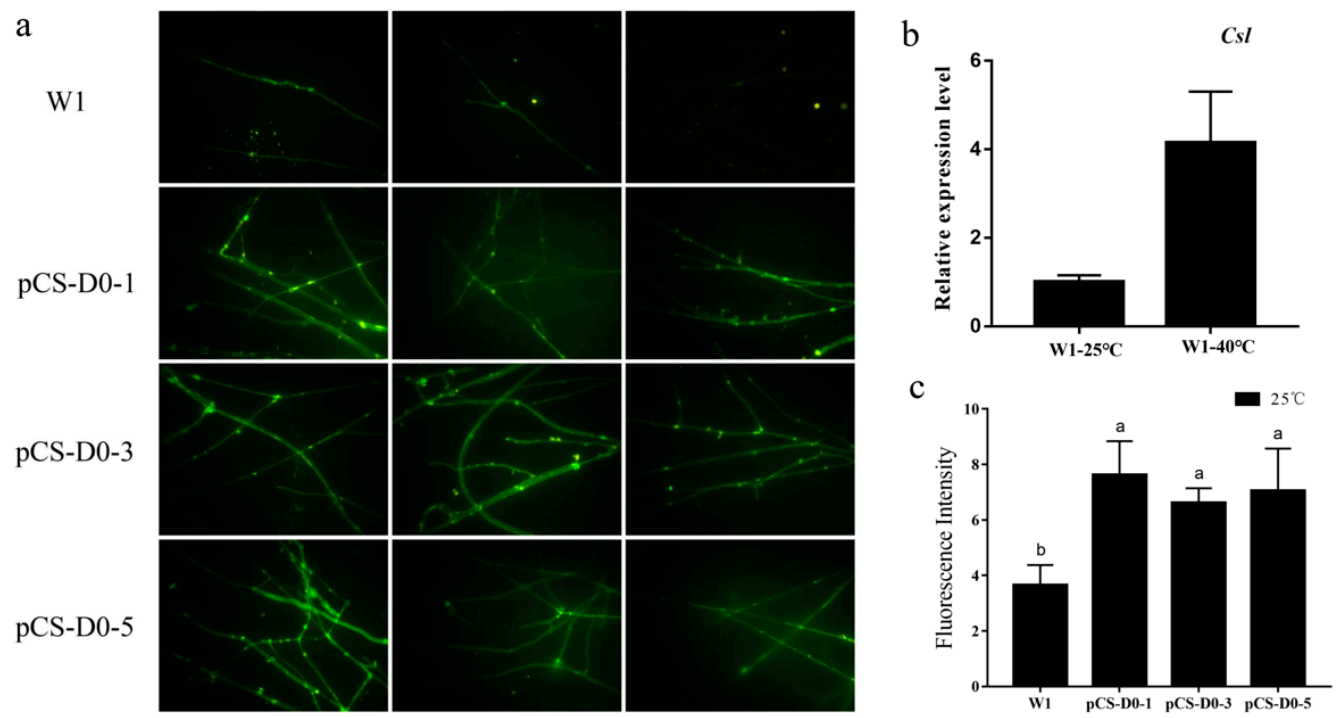

Figure 1. (a) Mycelia of pCS-D0 transformants observed with an OLYMPUS BX51 fluorescence microscope compared to wild-type W1; images were taken with a 40x field of view. (b) Relative expression level of Csl before and after heat stress in wild-type W1. (c) The fluorescence intensity of pCS-D0 transformants; $n=3, p<0.01$.

\subsection{Deletion Analysis of the $p C S$.}

To explore the heat-inducible element that responds to heat stress, a series of 5 'deletion constructs were undertaken and transferred into mycelia (W1). As shown in Figure 2, the wild-type W1 strain and pCS-D1,pCSD2, pCS-D3, pCS-D4 transformants showed no green fluorescence, but the fluorescence was obvious in $\mathrm{pCS}-\mathrm{D0}$, under normal cultivated condition $\left(25^{\circ} \mathrm{C}\right)$. The full-length fragment of $p C S$ (pCS-D0) showed the highest transcriptional activity, while the deletion fragment of $p C S$ did not improve the transcriptional activity significantly. Previous studies reported that the highly active fragment of GPD gene promoter of $795 \mathrm{bp}$ in Pleurotus ostreatus [20], $442 \mathrm{bp}$ in L. edodes [21], and $630 \mathrm{bp}$ in Aspergillus terreus [22], were more efficient than the full-length of GPD gene promoter. Therefore, we concluded that the deletion fragments of $p C S$ may not be more efficient than the full-length promoter.

After heat shock for $24 \mathrm{~h}$, the fluorescence intensity of pCS-D0, pCS-D1, and pCS-D2 transformant mycelia significantly increased; however, the pCS-D3 and pCS-D4 transformants showed no significant change. The results indicated that $p C S$ was an inducible promoter which can respond to heat stress and the heat-inducible element could be located between $-500 \mathrm{bp}$ and $-400 \mathrm{bp}$. The fluorescence intensity in all truncated constructs showed that the predicted HSTF binding sites, which were located between $+891 \mathrm{bp}$ and $+1015 \mathrm{bp}$ and $+102 \mathrm{bp}$ and $+497 \mathrm{bp}$, are not the functional element. Combined with the results of the bioinformatic analysis of the full-length $p C S$, two heat shock transcription factor (HSTF or Hsf1) binding domains were exactly located in this region $(-490 \mathrm{bp}$ to $-500 \mathrm{bp}$ and $+891 \mathrm{bp}$ to $+899 \mathrm{bp}$ ), but only one of them was predicted by SCPD and Yeastract simultaneously ( $-490 \mathrm{bp}$ to $-500 \mathrm{bp}$ ). Then, it was considered as the supposed heat-inducible element. 


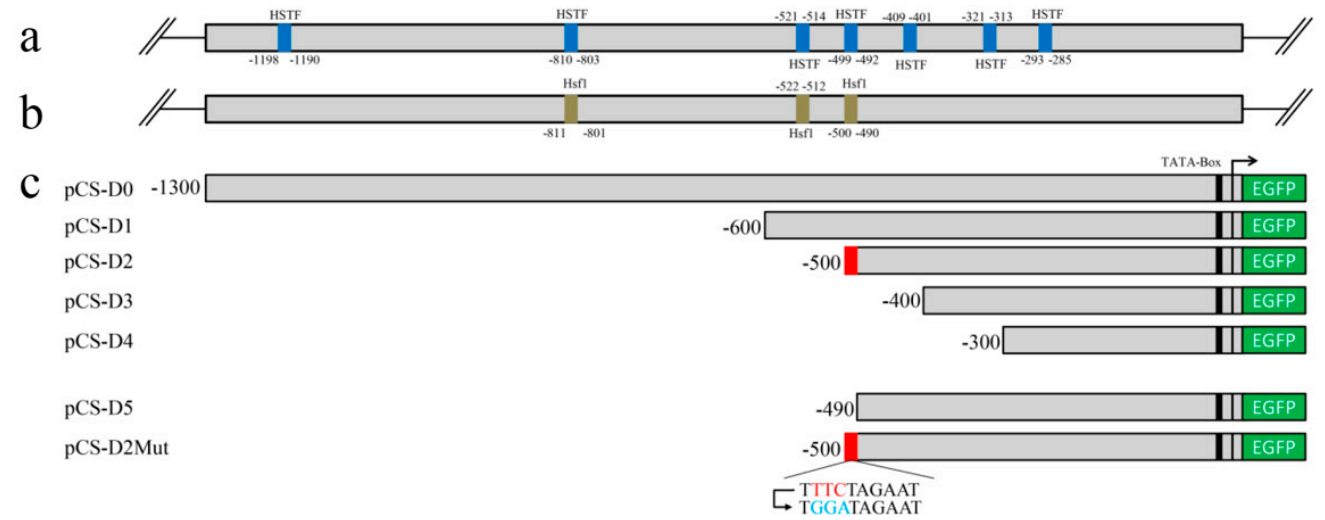

d
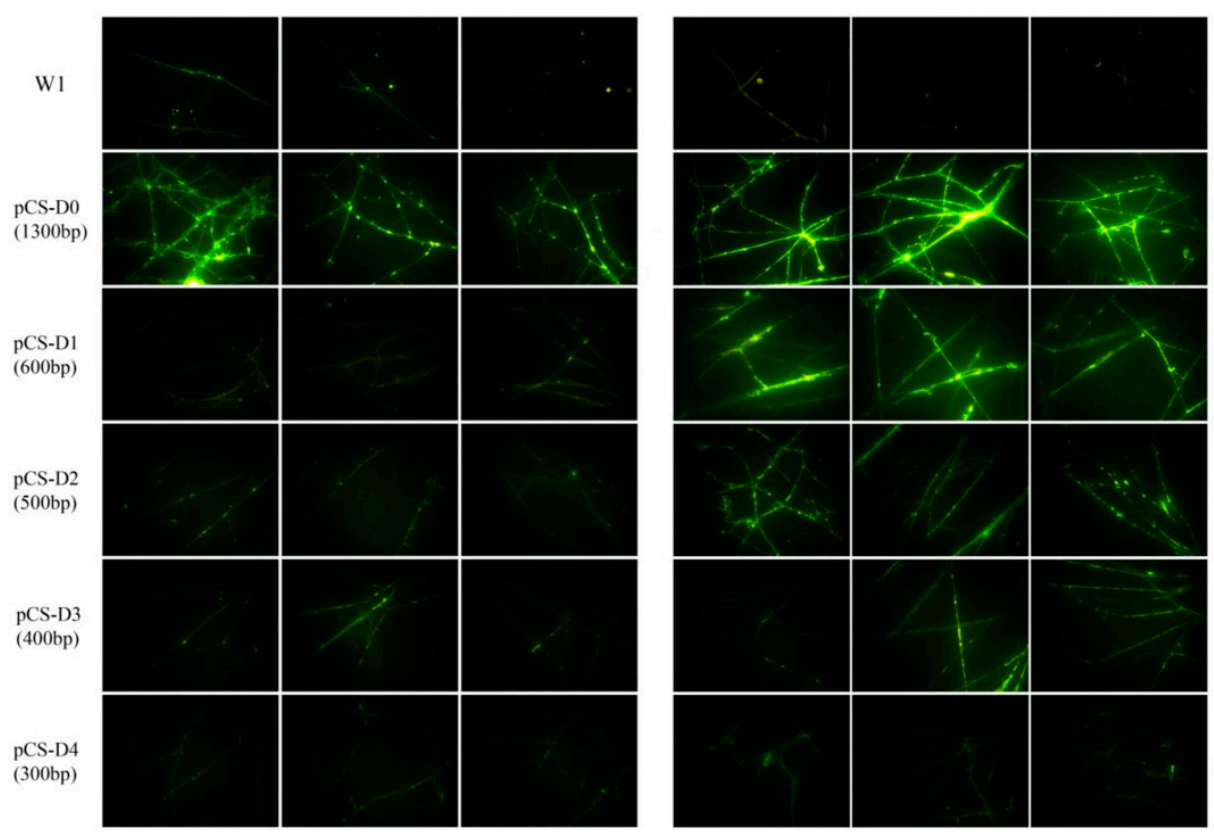

$\mathrm{e}$
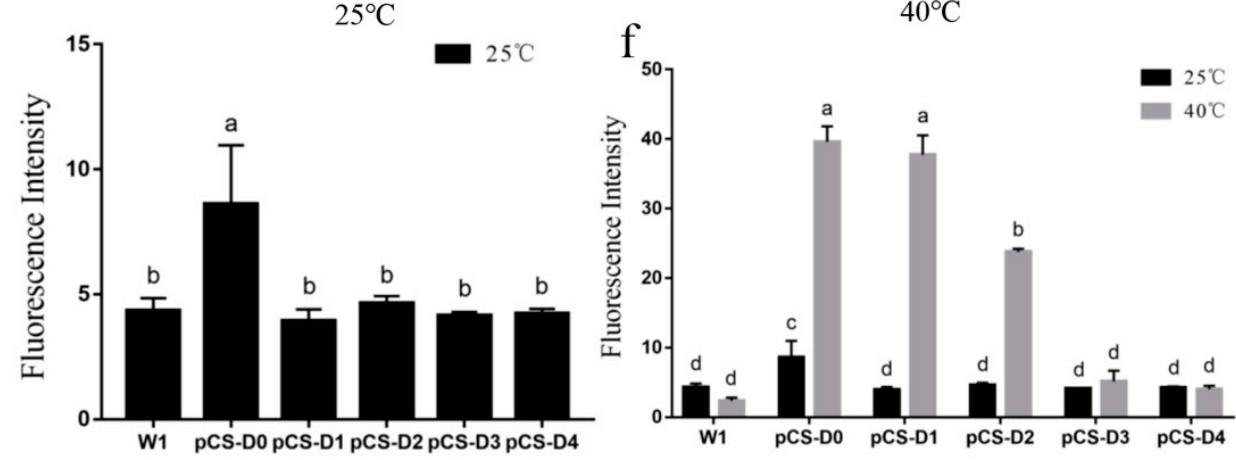

Figure 2. (a) HSTF binding sites in $p C S$ predicted by SCPD. (b) Hsf1 binding sites in $p C S$ predicted by Yeastract. (c) Construction of expression vectors with the full-length and truncated $p C S$ s. (d) Mycelia of different transformants before and after heat stress observed with an OLYMPUS BX51 fluorescence microscope compared to wild-type W1; images were taken with a $40 \times$ field of view. (e) The fluorescence intensity of different transformants under $25^{\circ} \mathrm{C} ; n=3, p<0.01$. (f) The fluorescence intensity of different transformants before and after heat stress; $n=3, p<0.01$.

\subsection{Site-Directed Mutation Analysis}

To verify the element within a $-490 \mathrm{bp}$ to $-500 \mathrm{bp}$ region that was responsible for heat shock, further truncation to $-490 \mathrm{bp}$ (pCS-D5) and mutation of this element (pCS-D2Mut) were conducted. As shown in Figure 3, the fluorescence intensity of pCS-D5 transformants mycelia showed no significant 
change after heat shock, while pCS-D2 transformants did, which meant the deletion of this element lead $p C S$ to fail to respond to heat stress. Meanwhile, the pCS-D2Mut transformants also showed no significant change. The difference between pCS-D2Mut and pCS-D2 was only three base-pairs in the supposed heat-inducible element, but completely changed the transcriptional activity. Generally, this $10 \mathrm{bp}$ between $-490 \mathrm{bp}$ and $-500 \mathrm{bp}$ (TTTCTAGAAT) was the key element required for heat response in $p C S$. Similar result was also reported for the HSE (heat shock element) in the HSP26 gene of Saccharomyces cerevisiae [23]. Moreover, the results also indicated that the one HSTF binding site located between +891 bp and +899 bp was not the functional element, which still appeared in the pCS-D5 and pCS-D2Mut constructs.

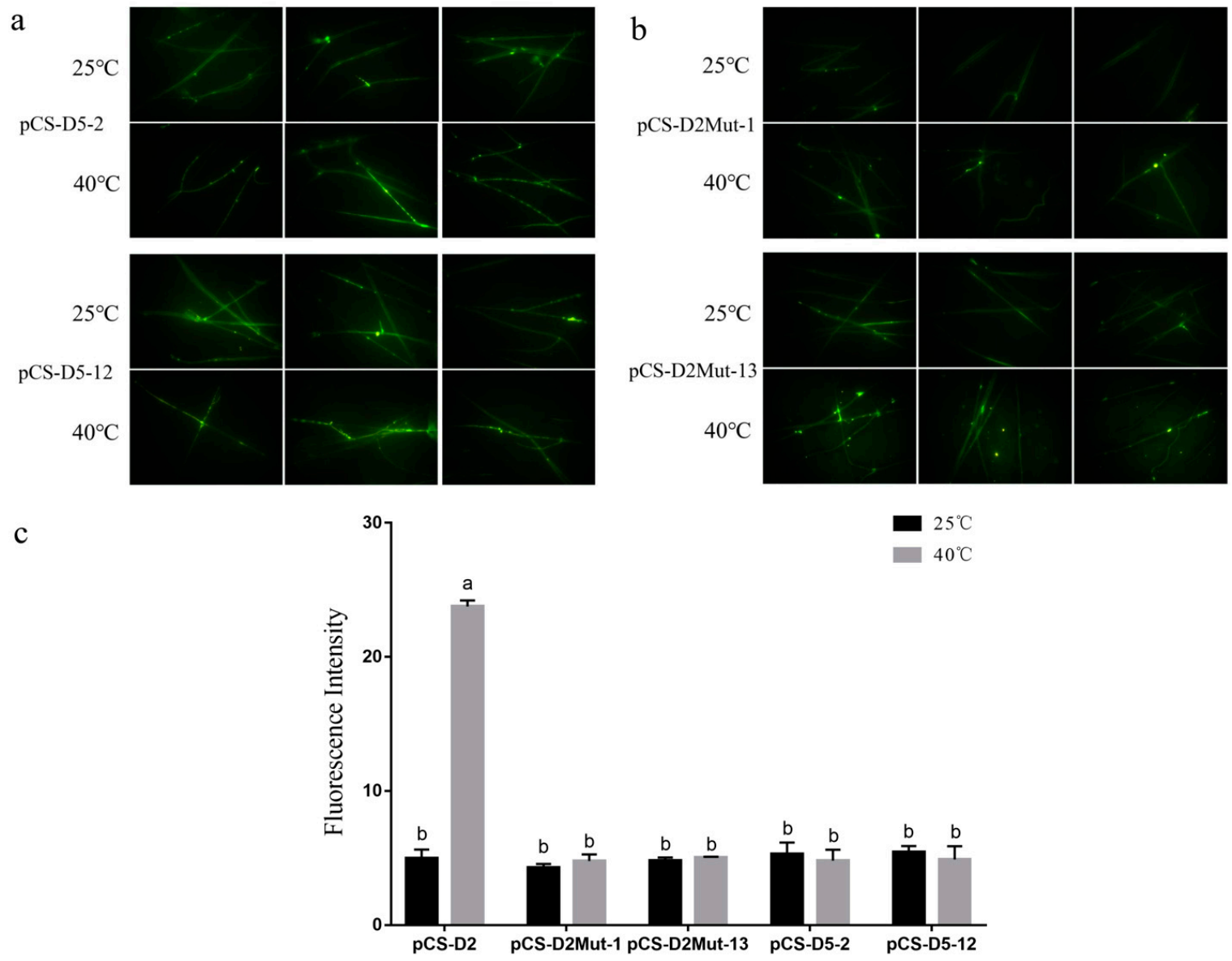

Figure 3. (a) Mycelia of pCS-D5 transformants before and after heat stress observed with an OLYMPUS BX51 fluorescence microscope; images were taken with a 40× field of view. (b) Mycelia of pCS-D2Mut transformants before and after heat stress observed with an OLYMPUS BX51 fluorescence microscope; images were taken with a $40 \times$ field of view. (c) The fluorescence intensity of pCS-D5 and pCS-D2Mut transformants before and after heat stress; $n=3, p<0.01$.

\section{Discussion}

Lentinula edodes is a delicious edible mushroom, with a unique volatile flavor, but the mechanisms of the volatile sulfide generation by heat stress is still at the protein level. The main contribution of this work is to advance the mechanisms of flavor formation at the molecular level. Combining the results of this paper, we speculate that the mechanisms of the volatile sulfide formation in the process of hot-air drying of L. edodes is as follows: In the early stage of the hot-air drying process, the water activity remains at a high level and the metabolic process still proceeds. It has been reported that the oxidative stress response was stimulated by high temperature during the early stage [24]. We suggest that cysteine desulfurase is activated by the oxidative stress because it is the first enzyme 
in the thiamine metabolic pathway while thiamine is closely related to the oxidative stress [25,26]. Thiamine metabolic pathway is activated to reduce the damage to mycelia under the oxidative stress. In this process, the HSTF binds with the heat-inducible element which locates between $-500 \mathrm{bp}$ and $-490 \mathrm{bp}$ in $p C S$ and enhances the transcriptional activity of the Csl gene, causing the accumulation of cysteine desulfurase. Subsequently, with the temperature rise and the water desorption, a large number of enzymatic reactions occur to generate volatile sulfide, including the reaction catalyzed by cysteine desulfurase with lentinic acid as the substrate. In the later stage of the drying process, non-enzymatic reactions such as a Maillard reaction occurs. Thiamine can degrade and generate volatile sulfide as well [27]. All these reactions contribute to the characteristic flavor for L. edodes. The proposed model of the formation of volatile sulfide generated by cysteine desulfurase during the hot-air drying process is shown in Figure 4.

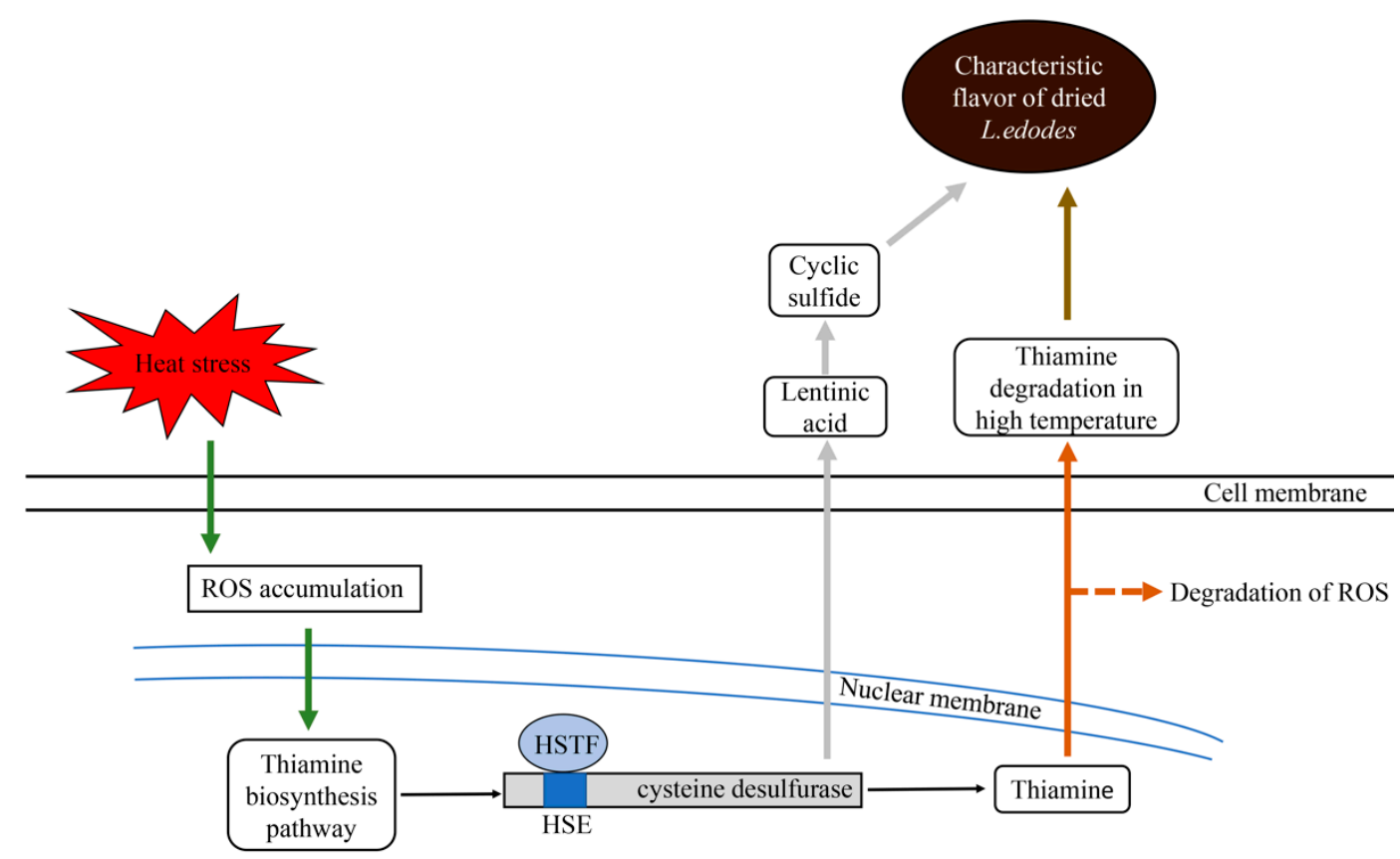

Figure 4. Schematic model of the formation of volatile sulfide during the hot-air drying process. The dotted arrows indicate the result is being explored. ROS (reactive oxygen species).

Additionally, the element between-500 bp and $-490 \mathrm{bp}$ was the functional heat-inducible element that responded to heat stress, and not the element which was located between, $-409 \mathrm{bp}$ and $-401 \mathrm{bp}$, $-321 \mathrm{bp}$ and $-313 \mathrm{bpand}-293 \mathrm{bp}$ and $-282 \mathrm{bp}$. The fluorescence intensity of pCS-D0 transformants after heat stress was 4.6-fold higher than before and 9.6-fold in pCS-D1, and 4.8-fold in pCS-D2. The fluorescence intensity was nearly the same in the pCS-D0 and pCS-D1 transformants after heat stress, but was significantly reduced in pCS-D1 under $25^{\circ} \mathrm{C}$. Therefore, these two elements $(-1198 \mathrm{bp}$ to $-1190 \mathrm{bp}$ and $-810 \mathrm{bp}$ to $-803 \mathrm{bp}$ ) possibly bound the HSTF during normal conditions to maintain the basal expression level of the Csl gene. Monnerjahn and Grosst reported that the HSF was also bound to HSE under normal conditions in Neurospora crassa, as well as Saccharomyces cerevisiae [28,29]. Furthermore, the predicted HSTF/Hsf1 binding site between $-522 \mathrm{bp}$ and $-512 \mathrm{bp}$ may be also a heat-inducible element which can bind with one HSTF and improve the transcriptional level of Csl after heat stress. It has yet to be further investigated in subsequent research work.

Interestingly, three coincident HSTF/Hsf1 binding domains and one HSTF binding domain (+102 bp to $+110 \mathrm{bp}$ ) shared the same signal sequence (NTTCNNGAAN), while the other three HSTF binding domains showed totally different signal sequence (GAANNNTCC) [30,31]. Previous studies indicated that HSTFs can bind DNA with the sequence NGAANNTTCN or with the sequence NTTCNNGAAN 
in yeast [32]. These two sequences are also called heat shock elements. Our research showed that the sequence of the elements in $p C S$ can respond to heat stress the same as the heat shock elements.

In $p C S$, there were three or six conceivable HSEs, but only one could respond to heat stress, which meant not all the HSEs can bind with HSTFs/Hsf1 or only several HSTFs/Hsf1 can bind with the promoter region. Young and Craig [33] found that the Hsp70 gene SSA1 had multiple heat shock elements, but only two of them were active promoter elements [33]. Chen and Pederson [23] also found that the rate of response to heat stress was correlated with the HSF occupancy of HSEs, rather than the number of HSEs in the promoter [23].

In sum, the heat-inducible element in the promoter $p C S$ can be a new target site for the flavor quality improvement and provide a deep understanding of the formation of flavor in L. edodes.

\section{Materials and Methods}

\subsection{Strains and Culture Conditions}

The wild-type strain L. edodes W1 (collected in Institute of Applied Mycology, College of Plant Science and Technology) used as the recipient host strain for transformation was grown on MYG medium ( $1 \%$ malt extract, $0.1 \%$ peptone, $0.1 \%$ yeast extract, $2 \%$ glucose) at $25^{\circ} \mathrm{C}$. The $\mathrm{DH} 5 \alpha$ strain of Escherichia coli used for plasmid amplification was grown on Luria-Bertani (LB) medium containing $100 \mu \mathrm{g} / \mathrm{mL}$ kanamycin at $37^{\circ} \mathrm{C}$. The EHA105 strain of Agrobacterium tumefaciens used for fungal transformation was grown on Luria-Bertani (LB) medium containing $100 \mu \mathrm{g} / \mathrm{mL}$ kanamycin and $50 \mu \mathrm{g} / \mathrm{mL}$ rifampicin.

\subsection{Extraction of Genomic DNA}

The mycelium was grown on cellophane membranes laid over MYG medium containing $5 \mu \mathrm{g} / \mathrm{mL}$ Hyg for 10 days at $25{ }^{\circ} \mathrm{C}$. Then, the mycelium was collected and ground to powder in liquid nitrogen. For DNA extraction, the mycelium powder was mixed with extraction solution including $475 \mathrm{uL}$ CTAB (Hexadecyl trimethyl ammonium Bromide, $100 \mathrm{mM}$ Tris- $\mathrm{HCl}(\mathrm{pH}=7.8), 20 \mathrm{mM}$ EDTA (Ethylenediaminetetraacetic acid), 1.4 M NaAc, 2\% (w/v) CTAB), and $25 \mu \mathrm{L} \mathrm{10 \%} \mathrm{SDS} \mathrm{(Sodium} \mathrm{dodecyl}$ sulfate) [34]. Then the tube was vortexed for $30 \mathrm{~s}$ and mixed with $500 \mu \mathrm{L} \mathrm{PCI} \mathrm{(Phenol:chloroform:isoamyl}$ alcohol $=25: 24: 1, v / v)$. After being centrifuged at 12,000 rpm for $10 \mathrm{~min}$, the supernatant was collected into a new centrifuge tube and mixed with $3 / 4$ volume isopropyl alcohol. Then, after being centrifuged at $12,000 \mathrm{rpm}$ for $5 \mathrm{~min}$, the precipitate was washed twice with $70 \%$ ethanol and dissolved with $100 \mu \mathrm{L}$ distilled water.

\subsection{Cloning and Analysis of Full-Length $p C S$}

According to the genome information of L. edodes v1.0 [35], we cloned the $1300 \mathrm{bp}$ up-stream of the initiation codon ATG in the Csl gene as the promoter region. The promoter sequence was amplified from the extracted genomic DNA of $L$. edodes $\mathrm{W} 1$ strain in a $50 \mu \mathrm{L}$ reaction system via PCR ( 3 min at $95^{\circ} \mathrm{C}, 35$ cycles of $15 \mathrm{~s}$ at $95^{\circ} \mathrm{C}, 15 \mathrm{~s}$ at $60{ }^{\circ} \mathrm{C}, 70 \mathrm{~s}$ at $72{ }^{\circ} \mathrm{C}$, and $5 \mathrm{~min}$ at $72{ }^{\circ} \mathrm{C}$ ) with paired primer pCS-R/pCSD0-F. This promoter region was analyzed online by SCPD (The Promoter Database of Saccharomyces cerevisiae, http://rulai.cshl.edu/SCPD/) and Yeastract (Yeast Search for Transcriptional Regulators and Consensus Tracking, http://www.yeastract.com/index.php) to predict the possible cis-acting elements as well as transcriptional factor binding sites.

\subsection{Vector Construction of the $p C S$ and Its Deletion Derivatives}

The full-length promoter and its 5 'deletion fragment were amplified from genomic DNA with primers in Table 3 via PCR reaction. The gene EGFP was amplified from the vector pCAMBIA1300-EGFP (constructed at the Institute of Applied Mycology, College of Plant Science and Technology) via PCR reaction. All PCR products were analyzed by agarose gel electrophoresis and collected with SanPrep 
Column DNA Gel Extraction Kit (Sangon Biotech, Shanghai, China). The pCAMBIA1300-g vector was digested with EcoR I and Kpn I in a $50 \mu \mathrm{L}$ reaction system. Then, the promoter fragment, EGFP, and the linearized pCAMBIA1300-g vector were linked together by homologous recombination to generate the promoter expression construct. For the mutation construct, the mutant fragment was simultaneously digested by EcoR I and Kpn I and then linked with linearized pCAMBIA1300-g backbone by T4 DNA ligase. Six promoter expression plasmids were then transformed into Agrobacterium tumefaciens strain EHA105.

Table 3. Sequence of the primers.

\begin{tabular}{|c|c|}
\hline Primer Name & Primer Sequence $\left(5^{\prime}-3^{\prime}\right)$ \\
\hline pCS-R & ccttgctcaccatGTTCAGTTAATCAAGGGGGTGAGG \\
\hline pCSD0-F & tctagaggatcccegggtaccATGGGTGAATATAGAGAGGCGG \\
\hline pCSD1-F & tctagaggatcccegggtaccCTGTAGCAGATTCTGAAAAGATTGTAGC \\
\hline pCSD2-F & tctagaggatccccgggtaccTTTCTAGAATCAGTTTGATTCAGGTCTG \\
\hline pCSD3-F & tctagaggatcccegggtaccTGAGATCTCATGCTACAGTGTGCA \\
\hline pCSD4-F & tctagaggatccccgggtaccAGGTAAGGAACTGTCCTTGATTTCA \\
\hline pCSD5-F & tctagaggatccccgggtaccCAGTTTGATTCAGGTCTGATTCGG \\
\hline EGFP-F & actgaacATGGTGAGCAAGGGCGAGG \\
\hline EGFP-R & ccacctcaaacttcggaattcTTACTTGTACAGCTCGTCCATGCC \\
\hline hph-F & TCGTCCATCACAGTTTGCC \\
\hline hph-R & TGCCTCTAATCCСTTGCTC \\
\hline qEGFP-F & AAGGGCATCGACTTCAAGGAG \\
\hline qEGFP-R & GTTCACCTTGATGCCGTTCTTC \\
\hline pCSD2Mut-F & GCCGAATTCTGGATAGAATCAGTTTGATT \\
\hline pCSD2Mut-R & GGTACCTTACTTGTACAGCTCGTCCAT \\
\hline
\end{tabular}

\subsection{Site-Directed Mutation and Further Truncation}

Based on the results of the truncated transformant mycelia fluorescence intensity as well as cis-acting element prediction of $p C S, 10 \mathrm{bp}$ (TTTCTAGAAT) located within $-490 \mathrm{bp}$ to $-500 \mathrm{bp}$ were suggested to be the conceivable element responding to heat stress. Then, the homologous recombination primers were designed to introduce $3 \mathrm{bp}$ sites mutation (TTTCTAGAAT changed into TGGATAGAAT) to this $10 \mathrm{bp}$. The forward primer contained a restriction enzyme cutting site of EcoR I and the reverse primer contained a site of Kpn I.

\subsection{Agrobacterium-Mediated Fungal Transformation}

Agrobacterium-mediated transformation of Lentinula edodes was performed as described by Wang Gang Zheng [34]. The A. tumefaciens strain EHA105 containing the promoter expression plasmid was cultivated at $28^{\circ} \mathrm{C}$ with shaking at $200 \mathrm{rpm}$ in MM (Minimal Medium) for 2 days. The Agrobacterium tumefaciens s cells were collected and suspended with IM (Induced Medium) to an OD600 around 0.4 and incubated for $6 \mathrm{~h}$ at $28{ }^{\circ} \mathrm{C}$ with shaking at $200 \mathrm{rpm}$. Mycelia of L. edoses were grown on a MYG plate for 2 days and then infected by pre-induced $A$. tumefaciens for 20 min and placed on Co-IM (co-cultivated induced medium) for 2 days at $28{ }^{\circ} \mathrm{C}$ to complete the fungal transformation. After 2 days of co-cultivation, the mycelia were transferred to selection medium containing $3 \mu \mathrm{g} / \mathrm{mL}$ hygromycin B and $200 \mu \mathrm{g} / \mathrm{mL}$ cefotaxime to screen the possible transformants, then continuously transferred the mycelia twice on selection medium containing hygromycin $\mathrm{B}$. The transformants were detected by PCR reaction using primers EGFP-F/R and hph-F/R to identify the band of the EGFP and $h$ hh genes, respectively.

\subsection{Fluorescence Microscopy and Quantification of EGFP Fluorescence Intensity}

Two positive L. edodes transformants were randomly selected and cultivated on MYG medium containing $5 \mu \mathrm{g} / \mathrm{mL}$ hygromycin B for 5 days until the mycelia were grown on the glass slides, while the wild-type W1 was cultivated without hygromycin B. The mycelia were observed under an OLYMPUS 
BX51 fluorescence microscope (OLYMPUS, Tokyo, Japan) with excitation at 455-490 nm to examine EGFP expression before and after heat shock $\left(40^{\circ} \mathrm{C}, 24 \mathrm{~h}\right)$. Images were taken with a $40 \times$ field of view. The imaged area was selected randomly, but clearly because the mycelia were grown irregularly. Each transformant was imaged in three different perspectives and the fluorescence intensity was the average value of these three perspectives. The numerical value of fluorescence intensity was calculated by Image-Pro Express (Media Cybernetics, Rockville, MD, USA). The $p$-value was calculated with the Duncan test and the error-bars in figures representing SD. The letters above the bars in figures represent different groups with highly significant differences $(p<0.01)$.

Author Contributions: Z.H., Y.L., and W.H. conceived and designed the experiments. X.L., S.G., and G.Z. prepared the experiment materials. Z.H. carried out the experiments and analyzed the data. Z.H., Y.L., and X.F. wrote the manuscript. Y.B., Y.L., and W.H. provided intellectual input and revised the manuscript. All authors read and approved the final manuscript.

Funding: This research was funded by the Natural Science Foundation of China (31601434), the Major Projects of Technological Innovation of Hubei Province (2017ABA148), the China Postdoctoral Science Foundation (2016T90701).

Conflicts of Interest: The authors declare no conflict of interest.

\section{References}

1. Liu, Y.; Yuan, Y.; Lei, X.; Yang, H.; Ibrahim, S.A.; Huang, W. Purification and characterisation of two enzymes related to endogenous formaldehyde in Lentinula edodes. Food Chem. 2013, 138, 2174-2179. [CrossRef] [PubMed]

2. Hiraide, M.; Yokoyama, I.; Miyazaki, Y. The smell and odorous components of dried shiitake mushroom, Lentinula edodes II: Sensory evaluation by ordinary people. J. Wood Sci. 2005, 51, 628-633. [CrossRef]

3. Chen, C.; Ho, C. Identification of sulfurous compounds of Shiitake mushroom (Lentinus edodes Sing.). J. Agric. Food Chem. 1986, 34, 830-833. [CrossRef]

4. Politowicz, J.; Lech, K.; Lipan, L.; Figiel, A.; Carbonell-Barrachina, Á.A. Volatile composition and sensory profile of shiitake mushrooms as affected by drying method. J. Sci. Food Agric. 2018, 98, 1511-1521. [CrossRef] [PubMed]

5. Chen, C.; Ho, C. High-performance liquid chromatographic determination of cyclic sulfur compounds of Shiitake mushroom (Lentinus edodes Sing.). J. Chrom. 1986, 356, 455-459. [CrossRef]

6. Hiraide, M.; Miyazaki, Y.; Shibata, Y. The smell and odorous components of dried shiitake mushroom, Lentinula edodes I: Relationship between sensory evaluations and amounts of odorous components. J. Wood Sci. 2004, 50, 358-364. [CrossRef]

7. Tian, Y.; Zhao, Y.; Huang, J.; Zeng, H.; Zheng, B. Effects of different drying methods on the product quality and volatile compounds of whole shiitake mushrooms. Food Chem. 2016, 197, 714-722. [CrossRef] [PubMed]

8. Yasumoto, K.; Iwami, K.; Mitsuda, H. Enzyme-catalized evolution of Lenthionine from Lentinic Acid. Agric. Biol. Chem. 1971, 35, 2070-2080. [CrossRef]

9. Yasumoto, K.; Iwami, K.; Mitsuda, H. Enzymic formation of Shiitake aroma from non-volatile precursor(s)-lenthionine from lentinic acid. Mushroom Sci. 1976, 9, 371-383.

10. Hiraide, M.; Kato, A.; Nakashima, T. The smell and odorous components of dried shiitake mushroom, Lentinula edodes V: Changes in lenthionine and lentinic acid contents during the drying process. J. Wood Sci. 2010, 56, 477-482. [CrossRef]

11. Liu, Y.; Lei, X.Y.; Chen, L.F.; Bian, Y.B.; Yang, H.; Ibrahim, S.A.; Huang, W. A novel cysteine desulfurase influencing organosulfur compounds in Lentinula edodes. Sci. Rep. 2015, 5, 10047. [CrossRef] [PubMed]

12. Liu, Y. Studies of the purification, cloning and biological function of key enzyme c-s lyase in the metabolism of endogenous formaldehyde in Lentinula edodes. Ph.D. Thesis, Huazhong Agricultural University, Huazhong, China, April 2014.

13. Gao, S.; Wang, G.; Huang, Z.; Lei, X.; Bian, Y.; Liu, Y.; Huang, W. Selection of reference genes for qRT-PCR analysis in Lentinula edodes after hot-air drying. Molecules 2018, 24, 136. [CrossRef] [PubMed]

14. Storozhenko, S.; De Pauw, P.; Van Montagu, M.; Inzé, D.; Kushnir, S. The heat-shock element Is a functional component of the Arabidopsis APX1 gene promoter. Plant Physiol. 1998, 118, 1005-1014. [CrossRef] [PubMed] 
15. Xiao, H.; Perisic, O.; Lis, J.T. Cooperative binding of drosophila heat shock factor to arrays of a conserved 5 bp unit. Cell 1991, 64, 585-593. [CrossRef]

16. Lockheart, A.; Curran, B.; Hirst, K.; Davies, M.W.; Piper, P.W.; Kingsman, A.J.; Stanway, C.A.; Ogden, J.E.; Kingsman, S.M. A heat shock element in the phosphoglycerate kinase gene promoter of yeast. Nucleic Acids Res. 1988, 16, 1333-1348.

17. ZUBER, U.; Schumann, W. CIRCE, a novel heat shock element involved in regulation of heat shock operon dnaK of Bacillus subtilis. J. Bacteriol. 1994, 176, 1359-1363. [CrossRef] [PubMed]

18. Uemura, H.; Koshio, M.; Inoue, Y.; Lopez, M.C.; Baker, H.V. The Role of Gcr1p in the Transcriptional Activation of Glycolytic Genes in Yeast Saccharomyces cerevisiae. Genetics 1997, 147, 521-532.

19. Peter, S.; Ayfer, B.; Marianne, K.; Thomas, N.; Klaus, S.; Joachim, M. Tec1p-independent activation of a hypha-associated Candida albicans virulence gene during infection. Infect. Immun. 2004, 72, 2386-2389.

20. Yin, C.; Zhu, J.; Ma, A.; Zheng, L.; Chen, L. Characterization of the highly active fragment of glyceraldehyde-3-phosphate dehydrogenase gene promoter for recombinant protein expression in Pleurotus ostreatus. Fems Microbiol. Lett. 2015, 362, fnv010. [CrossRef]

21. Cheng, S.; Yang, P.; Guo, L.; Lin, J.; Lou, N. Expression of multi-functional cellulase gene mfc in Coprinus cinereus under control of different basidiomycete promoters. Bioresour Technol. 2009, 100, 4475-4480. [CrossRef]

22. Huang, X.; Lu, X.; Li, J.J. Cloning, characterization and application of a glyceraldehyde-3-phosphate dehydrogenase promoter from Aspergillus terreus. J. Ind. Microbiol. Biotechnol. 2014, 41, 585-592. [CrossRef] [PubMed]

23. Chen, J.; Pederson, D.S. A distal heat shock element promotes the rapid response to heat shock of the HSP26 gene in the yeast Saccharomyces cerevisiae. J. Biol. Chem. 1993, 268, 7442-7448. [PubMed]

24. Wang, G.; Ma, C.; Luo, Y.; Zhou, S.; Zhou, Y.; Ma, X.; Cai, Y.; Yu, J.; Bian, Y.; Gong, Y. Proteome and transcriptome reveal involvement of heat shock proteins and indoleacetic acid metabolism process in Lentinula Edodes thermotolerance. Cell. Physiol. Biochem. 2018, 50, 1617-1637. [CrossRef] [PubMed]

25. Kowalska, E.; Kujda, M.; Wolak, N.; Kozik, A. Altered expression and activities of enzymes involved in thiamine diphosphate biosynthesis in Saccharomyces cerevisiae under oxidative and osmotic stress. FEMS Yeast Res. 2012, 12, 534-546. [CrossRef] [PubMed]

26. Medina-Silva, R.; Barros, M.P.; Galhardo, R.S.; Netto, L.E.S.; Colepicolo, P.; Menck, C.F.M. Heat stress promotes mitochondrial instability and oxidative responses in yeast deficient in thiazole biosynthesis. Res. Microbiol. 2006, 157, 275-281. [CrossRef]

27. Arnold, R.G.; Libbey, L.M.; Lindsay, R.C. Volatile flavor compounds produced by heat degradation of thiamine (vitamin B1). J. Agric. Food Chem. 1969, 17, 390-392. [CrossRef]

28. Monnerjahn, C.; Techel, D.; Meyer, U.; Rensing, L. Interaction of the Neurospora crassa heat shock factor with the heat shock element during heat shock and different developmental stages. Fems Microbiol. Lett. 2000, 185, 255-261.

29. Gross, D.S.; English, K.E.; Collins, K.W.; Lee, S.W. Genomic footprinting of the yeast HSP82 promoter reveals marked distortion of the DNA helix and constitutive occupancy of heat shock and TATA elements. J. Mol. Biol. 1990, 216, 611-631. [CrossRef]

30. Yang, W.M.; Gahl, W.; Hamer, D. Role of heat shock transcription factor in yeast metallothionein gene expression. Mol. Cell. Biol. 1991, 11, 3676-3681. [CrossRef]

31. Zhong, T.; Luke, M.T.; Arndt, K. Transcriptional regulation of the Yeast DnaJ homologue SIS1. J. Biol. Chem. 1996, 271, 1349-1356. [CrossRef]

32. J. Bonner, J.; Ballou, C.; Fackenthal, D. Interactions between DNA-bound trimers of the yeast heat shock factor. Mol. Cell. Biol. 1994, 14, 501-508. [CrossRef] [PubMed]

33. Young, M.R.; Craig, E.A. Saccharomyces cerevisiae HSP70 heat shock elements are functionally distinct. Mol. Cell. Biol. 1993, 13, 5637-5646. [CrossRef] [PubMed]

34. Wang, G.; Zhou, S.; Luo, Y.; Ma, C.; Gong, Y.; Zhou, Y.; Gao, S.; Huang, Z.; Yan, L.; Hu, Y.; et al. The heat shock protein 40 LeDnaJ regulates stress resistance and indole-3-acetic acid biosynthesis in Lentinula edodes. Fungal Genet. Biol. 2018, 118, 37-44. [CrossRef] [PubMed] 
35. Chen, L.; Gong, Y.; Cai, Y.; Liu, W.; Zhou, Y.; Xiao, Y.; Xu, Z.; Liu, Y.; Lei, X.; Wang, G. Genome Sequence of the Edible Cultivated Mushroom Lentinula edodes (Shiitake) reveals insights into lignocellulose degradation. PLoS ONE 2016, 11, e0160336. [CrossRef] [PubMed]

Sample Availability: Samples of the constructs are available from the authors.

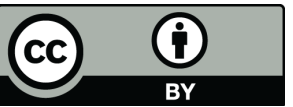

(C) 2019 by the authors. Licensee MDPI, Basel, Switzerland. This article is an open access article distributed under the terms and conditions of the Creative Commons Attribution (CC BY) license (http://creativecommons.org/licenses/by/4.0/). 